\title{
Tangence
}

\section{L'Apocalypse ou l'explosion de l'imaginaire}

\section{Jean-Pierre Prévost}

Numéro 35, mars 1992

Des écritures à lire

URI : https://id.erudit.org/iderudit/025697ar

DOI : https://doi.org/10.7202/025697ar

Aller au sommaire du numéro

Éditeur(s)

Tangence

ISSN

0226-9554 (imprimé)

1710-0305 (numérique)

Découvrir la revue

Citer cet article

Prévost, J.P. (1992). L'Apocalypse ou l'explosion de l'imaginaire. Tangence, (35), 35-45. https://doi.org/10.7202/025697ar d'utilisation que vous pouvez consulter en ligne.

https://apropos.erudit.org/fr/usagers/politique-dutilisation/ 


\title{
L'Apocalypse ou l'explosion de l'imaginaire
}

\author{
Jean-Pierre Prévost
}

\section{Un rendez-vous avec l'apocalypse}

Peu de livres bibliques peuvent revendiquer d'avoir alimenté l'imaginaire chrétien - et parfois de toute une société - autant que ne l'a fait l'Apocalypse de Jean. L'époque que nous vivons est particulièrement significative à cet égard, alors que la perspective d'une fin de millénaire suscite tout genre de spéculations, souvent accompagnées des prédictions les plus sombres.

Qu'il suffise d'en évoquer ici quelques manifestations. Dans les derniers jours de 1991, nous avons eu droit au rituel, maintenant coutumier, des prédictions attribuées aux astrologues, futurologues, et à qui sais-je encore? Dans ce concert de prédictions, où on fait la part belle à l'influence des astres et des planètes le passage à l'ère du Verseau apparaissant comme l'explication ultime -, le mot :apocalypse - refait facilement surface. Nous serions, paraît-il, la "génération de l'apocalypse *, ou pour employer les mots de Hal Lindsey, la "génération terminale ", avec tout ce que cela peut représenter de fatidique. C'est déjà ce que laissait entendre le cinéaste américain Francis Coppola, avec son fameux Apocalypse Now, qui voyait dans les lendemains de la guerre du Vietnam des airs de fin du monde. Reste à voir si cette lecture correspond à la réalité, la nôtre bien sûr, et aussi celle du livre biblique dont on se réclame.

L'interprète de chez nous Jo Bocan témoigne, elle aussi, de ce qu'on pourrait appeler la conscience apocalyptique, avec la chanson Apocalypso de son dernier album. Sans verser dans le fatalisme, elle fait état d'une préoccupation réelle, et le titre de sa chanson doit son pouvoir d'évocation en bonne part à l'Apocalypse de Jean.

Enfin, je retiens ici un autre indice de la popularité de l'Apocalypse dans l'imaginaire collectif. L'inquiétude apocalyptique a fait recette, aussi bien dans le domaine du cinéma que de la fiction littéraire, au cours des deux dernières décennies. Au cinéma, on ne compte plus les scénarios apocalyptiques: Le jour 
de la fin du monde, Apocalypse 2024, Le jour d'après, Holocauste 2000 , Pale Rider, etc. ${ }^{1}$ D'autre part, l'un des plus grands succès de librairie de la décennie a été le roman Le nom de la rose, d'Umberto Eco. Roman aux facettes multiples, cette œuvre de fiction repose essentiellement sur un fond d'apocalypse: on $y$ parle souvent de la Bête et la clef de l'intrigue policière se trouve dans la bibliothèque du fameux monastère. Or, les sections de cette bibliothèque sont marquées par des versets de l'Apocalypse! On ne sera donc pas surpris d'apprendre à la toute fin que l'incendie de l'Abbaye équivaut à la ruine de Babylone, décrite au chapitre 18 de l'Apocalypse. Et d'autres romans importants ont exploité la même veine, tels Le cinquième cavalier, de Lapierre et Collins, ainsi que Les bouffons de Dieu, de Morris West, qui comportent tous deux une citation de l'Apocalypse en exergue. On chercherait en vain une autre cuvre biblique qui ait inspiré, ces derniers temps, autant d'œuvres romanesques et cinématographiques. Bref, à bien des égards, nous avons rendez-vous avec l'apocalypse. Rendez-vous, par conséquent, avec l'imaginaire. Un imaginaire parfois peuplé des inquiétudes les plus redoutables, mais aussi un imaginaire qui entretient les plus belles espérances.

\section{L'Apocalypse, aux confluents de l'écriture et de la parole}

La Bible se situe aux confluents de l'écriture et de la parole. Reconnue par les croyants d'aujourd'hui comme la Parole de Dieu, elle est d'abord née d'une prise de parole humaine, fruit de l'écoute d'une autre Parole ou résultat de l'initiative de certains individus et des communautés qui les ont appuyés.

Mais la Bible est aussi, on le sait bien, une bibliothèque, et par conséquent, une littérature. Elle appartient au domaine de l'écrit, et ce n'est pas sans raison non plus qu'on l'appelle, sans autre déterminatif, l'Écriture.

Appartenant à cette imposante collection, l'Apocalypse de Jean en partage les deux traits essentiels et se situe, elle aussi, aux confluents de l'écriture et de la parole. Voyons d'abord

1 On trouvera une analyse détaillée de l'impact de ce thème sur la production cinématographique récente dans l'ouvrage d'H. Puiseux, L'apocalypse nucléaire et son cinéma, Paris, Cerf, coll. . $7^{\circ}$ Art*, $n^{\circ} 84,1987,235$ p. 
comment elle relève de la parole. Tout au long de l'Apocalypse, on trouve en effet différents niveaux de prise de parole.

Il y a d'abord la parole prophétique, imprévisible et tranchante, qui vient d'ailleurs, mais qui appelle des changements ici et maintenant. Les chapitres 2 et 3 , traditionnellement désignés comme *lettres * aux églises, appartiennent en effet au genre de l'oracle prophétique ("Ainsi parle...*) plutôt qu'au genre épistolaire, et appellent en conséquence une attitude d'écoute et de conversion: "Celui qui a des oreilles, qu'il entende ce que l'Esprit dit aux Églises . (2, 1 et parallèles). Toute l'attention porte sur l'autorité de celui qui parle et sur l'urgence de son message.

Dans le même sens, on retrouve la parole impérative, autoritaire et sans appel, des ordres adressés à l'auteur, à l'un des anges ou à un élément cosmique: ‘Écris! Viens! Attendez! Lève-toi! Jette ta faucille!... Parole qui retentit d'en haut, et qui suppose que l'histoire se décide à ce niveau-là aussi.

Mais cette parole échappe à la fatalité grâce aux interventions du visionnaire, qui chercbe à comprendre le sens des événements (par exemple, dans son dialogue avec l'un des vieillards, au sujet de l'identité des 144 000), et des martyrs, qui appellent le salut (6,10: *Jusques à quand, Maître saint et vrai, tarderas-tu à faire justice?.).

Et surtout, pour briser encore davantage le cercle de la fatalité, Jean nous met en présence d'une parole qui porte une profonde empreinte communautaire. En effet, les nombreux hymnes qui viennent ponctuer le livre (chapitres $4,5,7,11,12,15,19$ ) culminent sur un dialogue infini, où l'Épouse et l'Époux s'appellent inlassablement, comme jadis au temps du Cantique des cantiques. À l'invitation de l'Esprit et de l'Épouse: "Viens! ", le Christ répond par ces mots: : Oui, mon retour est proche! - $(22,17$ et 20). On est donc loin d'une création littéraire gratuite: toute l'œuvre vise à instaurer et à prolonger un dialogue entre le personnage central et les destinataires du livre, la communauté à laquelle Jean s'adresse.

\section{Le souci du livre et de l'écriture}

Si l'Apocalypse doit être entendue et reçue comme parole, elle s'impose aussi comme littérature. Elle est le fruit d'une écriture soignée et construite. Il faut retenir, en effet, avec André 
Paul, que .l'apocalypse, c'est d'abord de l'écriture et pas autre chose ${ }^{2}$. Une écriture prolifique, certes, qui s'est développée surtout en dehors de la Bible ${ }^{3}$, mais qui n'est pas inconnue non plus de la littérature biblique, avec des livres comme Ezéchiel et Daniel, hautement symboliques et nés, eux aussi, en période de crise.

Il existe bien quelques références au projet d'écriture dans la Bible: Ésaïe, Jérémie, Qohéleth, Luc-Actes, et certaines lettres de Paul. Mais c'est dans l'Apocalypse de Jean qu'on trouve exprimée le plus clairement et le plus fréquemment une allusion au livre et à l'entreprise littéraire. C'est ainsi, par exemple, que les chapitres 5 et 10 nous renvoient à l'image du livre scellé (chapitre 5) et du livre qu'il faut assimiler de l'intérieur (chapitre 10). D'autre part, Jean présente d'emblée son œuvre comme un écrit, en insistant sur sa réception du côté du lecteur: *Heureux celui qui lit, et ceux qui écoutent les paroles de la prophétie, et gardent ce qui s'y trouve écrit... * (1,3; cf. 22,7 et 10$)$.

On ne sera donc pas surpris d'entendre répéter, tout au long de l'œuvre johannique, l'ordre d'écrire (treize fois en tout, dont sept fois en introduction au message aux églises: $2,1.8 .12 .18 ; 3$, 1.7.14). À l'autre extrémité de son œuvre, on retrouve une cascade d'allusions au caractère littéraire du projet de Jean, puisque le mot * livre * y revient... sept fois $(22,7.9 .10 .18 .19)$ !

Deux autres passages viennent apporter un éclairage différent à cette thématique de l'écrit. D'une part, 19,9 confirme la ligne générale du livre, qui se veut une révélation. On y retrouve en effet une béatitude, et il s'agit bel et bien d'une parole venue d'ailleurs, paradoxale comme le sont les béatitudes, et transcendante au point de pouvoir être qualifiée de "paroles mêmes de Dieu ": :Un ange me dit: Écris! Heureux ceux qui sont invités au festin des noces de l'agneau! Puis il me dit: Ce sont les paroles mêmes de Dieu. " D'autre part, 10,4 fait état d'une certaine tension: le visionnaire s'apprête à écrire le contenu de ses visions,

2 A. Paul, - L'Apocalypse n'est pas la fin du monde, Etudes 355 (1981), p. 515.

3 On peut recenser une bonne trentaine d'apocalypses non bibliques sur une période de 350 ans, dans les siècles qui ont précédé ou suivi l'ère chrétienne. Voir à ce sujet le chapitre - Entrer dans le monde des apocalypses*, dans J.-P. Prévost, Pour lire l'Apocalypse, Novalis-Cerf, 1990, p. 65-82. 
sauf que tout ne doit pas être dévoilé: * Et quand les sept tonnerres eurent retenti, comme j'allais écrire, j'entendis une voix qui, du ciel, me disait: Garde secret le message des sept tonnerres et ne l'écris pas. * Voilà définie en quelques mots toute la problématique de l'Apocalypse. Essentiellement destinée à dévoiler, à communiquer, elle n'en demeure pas moins un écrit énigmatique, qui ne saurait représenter qu'une approximation du mystère.

Or là-dessus, l'Apocalypse de Jean rejoint l'une des caractéristiques de la littérature apocalyptique juive: celle d'être placée davantage sous le patronage des scribes (Hénoch, Baruch et Esdras), hommes de l'écrit, plutôt que sous celui des prophètes, hommes de la parole. Et si les auteurs d'apocalypses ne semblent refuser aucune ressource de l'imaginaire, leur souci de cohérence et de systématisation n'en est pas moins très marqué. C'est du moins ce que laissent entendre les titres génériques donnés à leurs œuvres: Livre ou Testament ${ }^{4}$. Il n'est d'ailleurs pas étonnant que les apocalypses soient apparues au moment où la Bible s'est formée comme livre, soit au temps de l'Exil (587-538): privé de ses institutions politiques, le peuple juif s'est regroupé autour de ses Écritures. La littérature venait de prendre *le relais de l'oracle $" 5$.

\section{Une œuvre construite}

Il ne manque pas d'entreprises ingénieuses pour essayer de présenter la structure de l'Apocalypse. Si les commentateurs ne s'entendent pas sur une structure définie, ce n'est pas faute d'indices, mais bien en raison d'un surplus. L'auteur a laissé plus d'une marque de son projet littéraire, et avec Jacques Ellul, il convient de parler d'. architecture en mouvement . 6 . Il y a à la fois cohérence et dynamisme. Les chapitres sont imbriqués les uns dans les autres et les parallèles abondent. La dynamique du livre repose en grande partie sur les correspondances qui existent entre les septs sceaux,

4 Parmi les apocalypses non bibliques, juives ou chrétiennes, ce sont les deux titres qu'on retrouve le plus fréquemment, après celui d'Apocalypse - apparu plus tardivement - : Livre des Jubilés, Liure étbiopbien d'Hénoch, 4' Livre d'Esdras, Livre des Secrets d'Hénoch, Testaments des Douze Patriarches, Testament de Moüse, Testament d'Abrabam.

5 A. Paul, L'Apocalypse n'est pas la fin du monde, Etudes 355 (1981), p. 518 .

6 J. Ellul, L'Apocalypse, arcbitecture en mouvement, Paris, Desclée, coll. - L'athéisme s'internoge *, 1975, 273 p. 
40

les sept trompettes et les sept coupes (chapitres 6-7; 8-11 et 16). Ces trois septénaires parlent tous de jugement et laissent entrevoir les signes de la fin, si bien qu'il ne faut pas les voir comme des événements distincts, mais comme une façon différente de percevoir la même réalité. On retrouve aussi un parallélisme marqué entre les deux livres (chapitres 5 et 10), encore que le premier apparaisse comme nettement plus décisif dans l'interprétation de l'histoire. L'auteur nous présente également les 144000 en deux situations fort diverses, soit au moment de l'épreuve (chapitre 7) et au moment de la délivrance et de la victoire (chapitre 14). Les lamentations sur la chute de Babylone (chapitre 18) feront place, quant à elles, aux réjouissances qui marqueront l'avènement de la Jérusalem nouvelle (chapitres 21-22).

Et enfin, chez Jean, le début et la fin se répondent admirablement, ce qui est sans doute déjà porteur d'un message: ce qu'on considère habituellement comme les événements de la "fin " est en fait un commencement, déjà rendu possible par la résurrection du Christ. C'est ainsi que la vision inaugurale $(1,9-20)$ et la vision finale $(22,6-20)$ se font écho, tandis que les promesses faites aux Églises (chapitres 2-3) anticipent sur plus d'un trait de la Jérusalem nouvelle (chapitres 21-22). Ce ne sont pas là deux mondes séparés, mais deux mondes qui s'appellent l'un l'autre.

Au-delà des ces correspondances, le caractère construit de l'œuvre se manifeste également par la symbolique établie par Jean, et dont la cohérence ne se comprend que par l'exercice littéraire qu'il s'est imposé.

C'est ainsi, par exemple, que les couleurs reçoivent une connotation bien précise. Le blanc est réservé à tout ce qui appartient au monde divin et au monde de la résurrection du Christ: le Fils de l'homme de la vision inaugurale a donc "les cheveux blancs. $(1,14)$, les vieillards qui entourent le trône céleste sont, comme le seront aussi les martyrs, "vêtus de blanc * $(4,4$ et 7,13$)$, le trône même de Dieu est blanc $(20,11)$, et le vainqueur associé à la résurrection du Christ se verra décerner un -caillou blanc. $(2,17)$. Ainsi en a décidé l'auteur: rien de ce qui est blanc n'appartient au monde de la Bête. Cette couleur est strictement réservée au monde divin, et en particulier au monde inauguré par le Christ ressuscité.

Quant au rouge, représenté en plusieurs nuances (rouge-sang, pourpre, écarlate), il est associé aux puissances sanguinaires et à 
la violence qu'ils exercent contre les saints $(6,4 ; 9,17 ; 12,3)$ ou à la débauche flamboyante des forces du mal $(17,3-4 ; 15,16)$. Le noir, bien que peu représenté (seulement en 6,5 et 6,12 ), reste affublé d'un air sombre et tragique, et demeure synonyme de malheur et de mort.

Oeuvre construite par son jeu de couleurs, l'Apocalypse l'est aussi, et de manière plus évidente et plus systématique encore, par l'utilisation des chiffres. Non pas que Jean ait innové sur toute la ligne. Il va puiser dans la symbolique biblique, en faisant appel à des chiffres déjà bien marqués, comme le sept, qui indique la plénitude, ou le douze, qui est la représentation idéale du peuple de Dieu, ou enfin le mille, que la Bible utilise sans scrupule mathématique pour désigner un grand nombre.

Fort de cette tradition, Jean a fait une répartition savante, certes, mais toujours cohérente, des chiffres. S'il nous parle donc des * sept églises ", il n'y a pas lieu de s'interroger pourquoi il s'est arrêté à sept, comme si les autres églises n'étaient pas importantes. Dire ssept églises", c'est s'adresser à toute l'Église de son temps, et même au-delà. Même chose pour ce qui est du déroulement ou du déploiement des sept sceaux, sept trompettes et sept coupes. Inutile de vouloir en établir une chronologie et d'en attendre l'accomplissement rigoureux, selon la séquence établie par l'auteur. Ce qu'il faut y voir plutôt, c'est une lecture globale des épreuves qui affligent déjà les contemporains de Jean. Celles qui sont énumérées ici ne sont pas exhaustives, mais exemplaires: elles représentent l'ensemble des tribulations présentes.

Quant au chiffre douze, il est toujours annonciateur de la destinée du peuple choisi, en référence directe aux douze tribus d'Israël $(7,1-8 ; 21,12)$ ou aux douze apôtres $(21,14)$. Dans le même sens, la Femme qui porte une couronne de - douze étoiles. $(12,1)$, doit être vue non pas comme une figure individuelle, mais comme le symbole du peuple de Dieu, engagé dans la lutte avec le Dragon, mais placé aussitôt sous le signe de la protection divine, qui lui assure un refuge au désert $(12,14)$.

Tout cela est manifestement le fruit d'une convention établie par l'auteur, et ce n'est qu'en prenant l'écrit dans son ensemble qu'on pourra interpréter les détails. Si l'œuvre n'était pas aussi construite, on pourrait peut-être en interpréter les différents éléments *à la pièce. Mais dans l'état actuel des choses, il faut recourir à l'ensemble pour en comprendre les parties. 


\section{Une écriture intertextuelle}

La Bible engendre la Bible. Le Deutéronome, comme son nom l'indique (du grec deutéros, deuxième, et nomos, loi) est une relecture de l'Exode, de même que les livres prophétiques viennent réactualiser la Loi consignée dans les cinq premiers livres de la Bible. Les psaumes engendrent les psaumes, et bon nombre de cantiques dispersés çà et là dans la Bible reprennent les refrains majeurs des psaumes. Le phénomène de l'intertextualité est omniprésent dans les évangiles, qui ne manquent jamais de souligner l'accomplissement des Écritures en général ou d'un oracle prophétique en particulier.

Le phénomène de l'intertextualité joue à plein dans l'Apocalypse de Jean. Celui-ci, en effet, est passé maître dans l'art de condenser les traditions bibliques et extra-bibliques. Pour ce qui est des traditions extra-bibliques, Jean puise abondamment dans l'arsenal de la littérature apocalyptique: même fascination pour le langage chiffré et les visions fantastiques, même sens de l'urgence du salut et du caractère dramatique, voire tragique, de l'histoire présente. Il excelle également à intégrer à ses réflexions de grands panneaux de la tradition biblique. Sans jamais épeler ses sources, Jean nous livre une puissante synthèse des grands courants de l'Ancien Testament.

Son livre est d'abord traversé par la perspective de l'Exode, avec au premier-plan le rappel de sept des dix plaies d'Égypte, et les thèmes récurrents de la révélation au Sinaï, de l'Agneau pascal, du cantique de Moïse, du séjour au désert, etc. Les épreuves auxquelles la communauté de Jean est confrontée rappellent donc pour lui la servitude et l'oppression dont le peuple a jadis souffert en Égypte. Et en utilisant tant de symboles de l'Exode, Jean veut nous faire comprendre qu'il est question ici d'une grande aventure de libération.

Les prophètes y occupent également une place de choix, surtout Ézéchiel (les quatre vivants, les quatre cavaliers, le petit livre avalé, la description du nouveau Temple et de la Jérusalem nouvelle, etc.), et Ésaïe (avec le rappel de la célèbre vision qu'il eut au Temple et qui fut accompagnée du -Saint, saint, saint *, et la vision du salut final, esquissée aux chapitres 65-66 d'Ésaïe). Et enfin, Jean utilise abondamment l'imagerie de Daniel: le fils de l'homme qui vient sur les nuées, les 1260 jours d'épreuve, la succession des royaumes, le culte impérial...). Jean est le digne 
successeur des prophètes qui naguère ont dénoncé les alliances illusoires et ont su ranimer l'espérance défaillante en temps de crise. Comme eux, il a le courage de dénoncer un pouvoir politique totalitaire et oppresseur. Et comme eux, il sait aller plus loin et convier le peuple à imaginer un avenir autre, à l'image du monde nouveau que Dieu veut façonner.

Autrement dit, l'Apocalypse de Jean est un livre qui a de qui tenir. Loin de représenter un phénomène isolé et déviant, il s'inscrit tout naturellement dans la tradition biblique. Certes, il en fait un condensé original, mais son imposante mosaïque nous renvoie immanquablement au coeur des Écritures. On pourrait donc parler d'une littérature au sujet d'une littérature.

\section{Un trésor de l'imaginaire}

Paradoxe des paradoxes: ce qui fait habituellement l'attrait de la littérature, soit le développement de l'imaginaire, est souvent apparu, dans le cas de l'Apocalypse comme une pierre d'achoppement. On reproche à l'auteur une sur-utilisation des symboles, que ce soit les animaux, familiers ou étranges (agneau, chevaux, dragon, bête à sept têtes et dix cornes, lions, sauterelles, scorpions, etc.), ou les chiffres (de un à deux cents millions, en passant surtout par le sept et le douze, et sans oublier le célèbre 666), ou encore les éléments cosmiques en processus de bouleversement profond: obscurcissement de la lune et du soleil, chute des astres, tremblements de terre, pollution des eaux, ravage des forêts, etc.

Par ailleurs, il suffit de se reporter à l'histoire de l'art chrétien pour se rendre compte que le livre de l'Apocalypse a été une source inépuisable d'inspiration pour les artistes chrétiens ${ }^{7}$. Des mosaïques romaines anciennes aux vitraux de Marc Chagall, en passant par les icônes orientales, les fresques romanes, la sculpture gothique, les enluminures et tapisseries médiévales, la peinture flamande et les gravures allemandes, on se rend compte que l'Apocalypse n'a jamais été confiné aux rayons d'une bibliothèque et à la simple lecture privée. C'est au contraire un livre public, ouvert à toutes les écoles et toutes les tendances.

7 Pour un inventaire des principales représentations artistiques de l'Apocalypse, $c f$. J. Hadot, Apocalypse de Jean dans l'art., Encyclopaedia Universalis, t. 2, Paris, 1984, p. 366-370 (avec références bibliographiques). 
Quoi de plus riche, en effet, que ce livre qui nous présente le Christ comme celui qui tient le glaive acéré dans sa bouche, et dans sa main les sept étoiles, représentant les églises? ou encore comme l'Agneau? ou l'étoile du matin? Quoi de plus évocateur, pour une assemblée liturgique, que les gestes de prière et d'adoration des vingt-quatre vieillards devant le trône céleste? quoi de plus frappant, dans l'imagination populaire, que les anges aux trompettes et aux coupes, annonçant l'imminence du jugement? et comment ne pas être éblouis par la beauté édénique de l'Arbre de vie et par l'éclat de la Jérusalem nouvelle, ornée de pierre précieuses et illuminée par le soleil de Dieu et de l'Agneau? Et que dire des anti-héros de l'Apocalypse - la Bête aux sept têtes et aux dix cornes, le Dragon rouge-feu et la Prostituée aux atours aguichants et trompeurs? Leurs attributs n'ont pas manqué non plus d'influencer les représentations des forces du mal à travers l'histoire de l'art.

On s'incline devant tant de richesses et tant de puissance symbolique. Fresque immense, l'Apocalypse demeure un livre visuel, véritable fête pour les yeux. Avec Jean, il faut s'empresser de regarder et de voir. L'œuvre de Jean se donne à contempler, et non à calculer. Il ne faut pas en faire un champ de bataille, ni de prédictions chiffrables, mais plutôt l'accueillir comme un appel à voir plus loin et plus profond.

\section{La « clôture * des Écritures}

Enfin, il ne faut pas oublier la position privilégiée que l'Apocalypse occupe dans la Bible. Dans l'aménagement de la Bible chrétienne, c'est ce qui vient conclure l'ensemble des écrits considérés comme inspirés. De ce fait, les derniers mots de l'Apocalypse ne viennent pas seulement mettre le point final aux propos de Jean mais ils viennent, ainsi que tout le livre de l'Apocalypse donner une coloration nouvelle à l'ensemble des Écritures. Désormais, la lecture ne peut se passer de cette clef apocalyptique. L'Apocalypse illumine tout ce qui précède et ouvre au mystère que les Écritures recèlent.

On ne pouvait trouver meilleure conclusion à l'ensemble des Écritures. Ce n'est pas par accident que la Bible se termine par un livre dont le titre veut dire "révélation", ce qui est vrai de l'ensemble de la collection dont l'Apocalypse fait partie. Et c'est aussi l'apocalypse de Jésus-Cbrist, pour confirmer que les Écritures ont 
nécessairement une dimension christologique, ou messianique. Tout, y compris l'histoire à venir, doit être interprété à partir de cette figure centrale, désormais inséparable du visage historique de Jésus de Nazareth.

L'écriture de l'Apocalypse signifie donc la fin d'une littérature, mais non de l'histoire. La vision que l'Apocalypse de Jean nous livre fait appel à la vigilance - et donc à la responsabilité - et au courage. Puisque les derniers mots de ce livre sont un dialogue et expriment une tension vers le retour du Christ ressuscité, il faut jouer à plein la carte de l'histoire. Tout comme les premières pages de la Genèse ne prétendent aucunement donner le dernier mot sur le comment de l'apparition de la vie, les dernières pages de la Bible chrétienne ne sauraient dispenser d'un engagement dans l'histoire. L'Apocalypse de Jean marque bien plus la fin d'une littérature que la fin du monde et la fin de l'histoire. C'est pour cela que tant de générations avant nous ont eu recours à ce livre, et il est permis d'espérer - sans avoir aucune certitude - que ce sera le cas pour bien d'autres générations après nous. Pourquoi pas? 